\title{
Multi-Hop Diversity Aided Multi-Hop Communications: A Cumulative Distribution Function Aware Approach
}

\author{
Dong Chen, Lie-Liang Yang, Senior Member, IEEE, and Lajos Hanzo, Fellow, IEEE \\ School of ECS, University of Southampton, SO17 1BJ, UK \\ E-mail: cd2g09,1ly,lh@ecs.soton.ac.uk, http://www-mobile.ecs.soton.ac.uk/
}

\begin{abstract}
In this contribution, we propose and study a signal transmission scheme, namely the multihop diversity (MHD) scheme, conceived for transmission of information over multihop links (MHLs). During each time-slot (TS), the MHD scheme activates the specific hop transmission, whose signal-to-noise ratio (SNR) cumulative distribution function (CDF) gives the highest ordinate value amongst all the available hops. The next packet is then transmitted over the selected hop. Our studies show that this CDF-aware MHD scheme represents a generalized MHD scheme suitable for operation in the scenarios, where the different hops may have different distances, hence resulting in different average SNRs, and/or experience different types of fading. This MHD scheme is also capable of achieving the full diversity gain provided by the independent fading experienced by the different hops. In this paper, our attention is dedicated to the error probability, outage probability as well as to the achievable diversity order, when BPSK and $M$-ary quadrature amplitude modulation (MQAM) are employed. Both the error probability and the outage probability of the MHLs are investigated for transmission over Nakagami- $m$ fading channels, both of which demonstrate a significant diversity gain. Furthermore, the impact of the various system parameters on the achievable error probability and outage performance is characterized.
\end{abstract}

Index Terms-Multihop communication, cumulative distribution function, diversity, relay, Nakagami- $m$ fading channel, error probability, outage probability, diversity order.

\section{INTRODUCTION}

In multihop wireless communications, the source nodes (SNs) send information to the corresponding destination nodes (DNs) via intermediate relay nodes (RNs), which provides a range of advantages over conventional single-hop communications. These advantages may typically include an improved link performance, quantified in terms of energy-efficiency and extended coverage, enhanced throughput, simplicity and flexibility of network planning, etc [1-3]. Hence, multihop networks have attracted much attention, as evidenced by [111] and the references therein.

In the context of multi-hop links (MHLs), it has typically been assumed that information is successively transmitted from the SN to the DN node by node, without any store-andwait stage at the intermediate RNs $[4,5,8]$. For convenience

The financial support of the RC-UK under the auspices of the India-UK Advanced Technology Centre, the support of European Research Council's Advanced Fellow Grant, the support of the EU's Concerto project and the UK/China scholarships for excellence programme is gratefully acknowledged. This paper was presented in part at the IEEE Global Telecommunications Conference, Houston, Texas, USA, December 2011. of description, we refer to this scheme as the conventional multi-hop transmission scheme in our forthcoming discourse. In this conventional multi-hop scheme, information is transmitted over a single hop during its scheduled time-slot (TS), regardless of its link quality often quantified, for example, by its signal-to-noise ratio (SNR). Hence, the overall reliability of a MHL is dominated by that of the poorest hop and a routeoutage occurs, once a hop outage is encountered in any of the invoked hops. As a result, the route's BER and outage performance of a MHL typically degrades or remains the same in the best case, as the number of hops increases. In order to improve the performance of MHLs, novel signaling schemes have been proposed $[1,3,12]$, which require the nodes to have a store-and-wait capability. For example, in [1, 12], adaptive modulation and coding (AMC) combined with automatic repeat request (ARQ) schemes has been invoked in cooperative decode-and-forward (DF) communications. Recently, the authors of [3] have employed AMC for dual-hop cooperative communications relying on a regenerative $\mathrm{RN}$, where the AMC mode of both the hops may be configured independently. Very recently, the buffer-aided transmission scheme was exploited in a two hop network $[13,14]$.

In [15-17], we have proposed a multihop diversity (MHD) scheme by exploiting the independent fading experienced by the different hops of a MHL. The studies demonstrated that significant performance improvements may be attainable in comparison to the conventional multi-hop transmission scheme. In [15-17], we assumed that all hops have the same distance and also experience the same fading CDF. Under these assumptions, during each TS, the specific hop having the highest instantaneous SNR was selected from the set of available hops and, then a packet was transmitted over the selected hop. Explicitly, this MHD scheme achieved selection diversity. Furthermore, all the hops have the same probability to be chosen during a TS, which is desirable for attaining the maximum possible throughput and minimum possible transmission delay.

As a further development of our work in [15-17], in this contribution, we generalize both the system model and the corresponding MHD scheme studied in [15-17]. In the context of the system model, we now consider the general MHLs, where different hops may have different distances and may also experience different fading. When selecting a hop for transmission based on the instantaneous SNRs of the hops 
invoked, as in [15-17], it will result in the hops being selected with different probabilities. Explicitly, a hop having a higher average SNR owing to experiencing less severe fading has a higher chance of being activated than a hop having a lower average SNR. Clearly, this is undesirable. In order to circumvent this impediment, while still benefitting from MHD, our MHD scheme proposed in this contribution opts for activating a hop for transmission according to the instantaneous probability values provided by the SNR's cumulative distribution functions (CDFs) of the available hops. To be more specific, let us assume that $L$ hops are available for selection, where we assume having the knowledge of the $L$ Nakagami$m$ parameters of $m_{1}, m_{2}, \ldots, m_{L}$ and the knowledge of the $L$ SNR values of $\gamma_{1}, \gamma_{2}, \ldots, \gamma_{L}$. Therefore, the SNR CDF ordinate values of $F_{m_{l}}\left(\gamma_{l}\right), l=1,2, \ldots, L$, can be calculated. Then, the hop having the highest CDF ordinate value is selected for transmitting a packet within the TS. This way we can avoid granting a higher transmission probability to the links subjected to benign fading. To elaborate a little further, activating always the highest-SNR link would potentially lead to buffer-overflow for the severely fading lower-SNR links. More explanation, it is possible that a higher SNR $\gamma_{l}$ actually maps to a lower $F_{m_{l}}\left(\gamma_{l}\right)$ ordinate value, even if the fading parameter $m_{l}$ is higher, because the fading is less severe. In this way, it can be shown that all $L$ hops have the same probability to be chosen, regardless of the specific fading parameter $m_{l}$ experienced by them. Note however that if all hops have the same average SNR and also experience the same fading CDF, implying that all hops have the same SNR $\mathrm{CDF}$, our CDF-assisted MHD scheme proposed in this paper becomes equivalent to the MHD scheme considered in [1517]. This is, because in this case a higher instantaneous SNR generates a higher CDF value.

In this paper, we study the performance of the MHLs employing our proposed MHD scheme, when assuming that the different hops may experience different fading CDF modeled by Nakagami- $m$ distributions associated with different fading parameters. We analyze both the BER and outage probability of the MHLs employing $M$-ary quadrature amplitude modulation (MQAM), when either buffers of limited or unlimited size are used. We derive both the lower-bound expression and the exact expressions for them. Furthermore, approximation technologies are proposed for the efficient evaluation of both the BER and of the outage probability. Additionally, we demonstrate that our proposed MHD scheme is capable of achieving the full multi-hop diversity. Our performance results demonstrate that the CDF-assisted MHD scheme has the potential of providing a significant diversity gain for improving the reliability of multihop communications, hence both the attainable BER and the outage performance can be enhanced.

We should note that, the terminology of MHD has also been used in [18]. However, the MHD considered in [18] and that defined in this paper as well as in [15-17] have an entirely different meaning. Specifically, in [18] it is assumed that a receiver node can receive replicas of the same signal from several other nodes. In this case, MHD is achieved at the receiver node by combining the signals received from the different nodes transmitting the same information. Therefore, the MHD in [18] is obtained by multipath combining. By contrast, our MHD belongs to the category of a type of selection diversity. Furthermore, in [18], the RNs do not use a buffer for storing packets, hence the signals are transmitted hop-by-hop based on the conventional multi-hop transmission scheme.

Additionally, we noted that as analyzed in [16], once a SN completes its transmission, an increased buffering-induced delay is imposed, since the RNs have to empty their buffer. This process makes the overall delay higher than that of the conventional multihop transmission scheme [19]. However, for the transmission of a sufficiently large amount of data, the multihop diversity scheme does not have to strike an explicit trade-off between the delay tolerated and the achievable error/outage performance on average, because the MHD scheme transmits a single packet over a single hop per time-slot, which is identical to the conventional multihop transmission scheme.

The contributions of this paper are summarized as follows:

1. A CDF-assisted MHD scheme is proposed, which makes use of the multiple hops of a MHL for enhancing the reliability of information delivery over the MHL.

2. The end-to-end BER and end-to-end outage probability of MHLs are analyzed, when the signal is transmitted using $M Q A M$, leading to a range of expressions for both the lowerbound and for the exact BER as well as for the lower-bound and the exact outage probability.

3. An approximate algorithm is provided for evaluating the steady-state probabilities that are required for computing both the end-to-end BER and the end-to-end outage probability.

4. We demonstrate that for an L-hop link, the achievable diversity order is $L$.

The remainder of this paper is organized as follows. In the next sections, we present both our system model and the implementation of the MHD scheme. Section III analyzes both the BER and the outage probabilities. In Section IV, we provide our numerical and simulation results. Finally, our conclusions are offered in Section V.

\section{System Model of Multi-Hop LinKS}

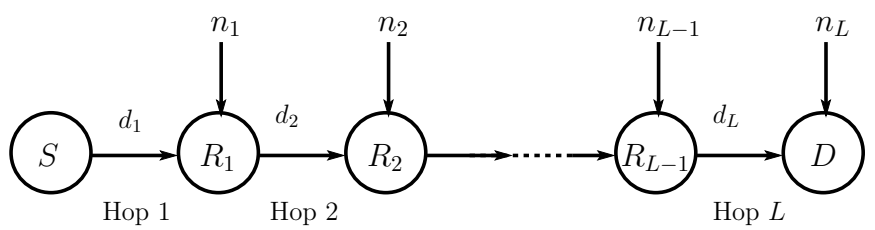

Fig. 1. System model for a multihop wireless link, where the source $S$ sends messages to the destination $D$ via $(L-1)$ intermediate relays.

The system model under consideration is a typical multihop wireless link $[8,19]$, which is shown in Fig. 1. The MHL consists of $(L+1)$ nodes, a SN $S$ (node 0$),(L-1)$ RNs $R_{1}, R_{2}, \cdots, R_{L-1}$ and a DN $D$ (node $L$ ). The distance of the $l$ th hop between nodes $(l-1)$ and $l$ is $d_{l}, l=1, \cdots, L$, and the total distance or the distance between $\mathrm{SN}$ and DN is $d=\sum_{l=1}^{L} d_{l}$. The SN $S$ sends its information to the DN $D$ via $L$ hops with the aid of the $(L-1)$ RNs. At the RNs, the classic 
decode-and-forward (DF) protocol is employed for relaying the signals. For convenience, we denote the symbol transmitted by node 0 as $x_{0}$ and its estimate at the DN $D$ of node $L$ by $x_{L}=\hat{x}_{0}$, while the symbol estimated at the $l$ th $\mathrm{RN}$ by $x_{l}, l=1, \ldots, L-1$. At packet level, they are correspondingly represented by $x_{0}, x_{l}, l=1, \ldots, L-1$, and $\boldsymbol{x}_{L}=\hat{\boldsymbol{x}}_{0}$. We assume baseband BPSK/MQAM modulation and that the signals are transmitted on the basis of TSs having a duration of $T$ seconds. In addition to propagation pathloss, the channels of the $L$ hops are assumed to experience independent block-based flat Nakagami- $m$ fading, where the complex-valued fading envelope of a hop remains constant within a TS, but it is independently faded for different TSs. Based on the above assumptions, when the $(l-1)$ st node transmits a packet $\boldsymbol{x}_{l-1}$, the observations received by node $l$ can be expressed as

$$
\boldsymbol{y}_{l}=h_{l} \boldsymbol{x}_{l-1}+\boldsymbol{n}_{l}, l=1,2, \ldots, L,
$$

where $h_{l}$ represents the channel gain of the $l$ th hop from node $(l-1)$ to node $l$, while $\boldsymbol{n}_{l}$ denotes the Gaussian noise at node $l$. When communicating over Nakagami- $m$ fading channels, $\left|h_{l}\right|$ obeys the Nakagami- $m$ distribution with the probability density function (PDF) [20]

$$
f_{\left|h_{l}\right|}(y)=\frac{2 m_{l}^{m_{l}}}{\Gamma\left(m_{l}\right)} y^{2 m_{l}-1} \exp \left(-m_{l} y^{2}\right), y \geq 0
$$

where $\Gamma(\cdot)$ denotes the gamma function [21](8.310.1), $E\left[\left|h_{l}\right|^{2}\right]=1$ and $m_{l}$ is the Nakagami- $m$ fading parameter of the $l$ th hop ${ }^{1}$. As shown in [25], the Nakagami- $m$ fading can be used for modelling of different types of fading channels, which are characterized by the associated fading parameter $m$. Specifically, the Nakagami- $m$ fading reduces to the Rayleigh fading when $m=1, m \rightarrow \infty$ corresponds to the conventional Gaussian scenario, and $m=1 / 2$ describes the so-called oneside Gaussian fading, i.e., the worst-case fading condition. The Rician and lognormal distributions can also be closely approximated by the Nakagami distribution in conjunction with values of $m>1$. In (1), the background noise samples of $n_{l}$ obey the complex Gaussian distribution with zero mean and a variance of $\sigma_{l}^{2}=1 /\left(2 \bar{\gamma}_{l}\right)$ per dimension. Here, $\bar{\gamma}_{l}$ denotes the average SNR of the lth hop, which is dependent on the propagation pathloss experienced by the $l$ th hop.

Let us consider a given TS. The corresponding instantaneous SNR values of the $L$ hops are expressed as $\left\{\gamma_{1}, \gamma_{2}, \ldots, \gamma_{L}\right\}$, where $\gamma_{l}=\left|h_{l}\right|^{2} \bar{\gamma}_{l}, l=1, \ldots, L$. Then, given the PDF of $\left|h_{l}\right|$ as shown in (2), the PDF and CDF of $\gamma_{l}$ can be readily derived, which are

$$
\begin{aligned}
& f_{l}\left(\gamma_{l}\right)=\frac{1}{\Gamma\left(m_{l}\right)}\left(\frac{m_{l}}{\bar{\gamma}_{l}}\right)^{m_{l}} \gamma_{l}^{m_{l}-1} \exp \left(-\frac{m_{l} \gamma_{l}}{\bar{\gamma}_{l}}\right), \gamma_{l} \geq 0 \\
& F_{m_{l}}\left(\gamma_{l}\right)=\frac{\gamma\left(m_{l}, \frac{m_{l} \gamma_{l}}{\bar{\gamma}_{l}}\right)}{\Gamma\left(m_{l}\right)}, \gamma_{l} \geq 0
\end{aligned}
$$

\footnotetext{
${ }^{1}$ The estimation of parameter $m$ is important for practical systems. A related paper [22] and the references cited in it discussed this issue. The fading parameter can be estimated according to (6) of [22] and the result is accurate within an average mean squared error of $3.81 \times 10^{-5}$. Apart from the model mentioned in this paper, both Kronecker model [23] and the Weichselberger model [24] are capable of accurately estimating the channel parameters at a low complexity for SISO system.
}

where $\gamma(a, x)$ is the incomplete gamma function [26].

Given the SNRs, $\gamma_{1}, \gamma_{2}, \ldots, \gamma_{L}$, of the $L$ hops within a TS, in our multi-hop transmission scheme, the packets are transmitted over the MHLs based on the following strategy. Among those hops, whose transmitter buffers have packets awaiting transmission and whose receivers are ready for reception, the MHD protocol first decides, which of the $L$ CDFs, $F_{m_{1}}\left(\gamma_{1}\right), F_{m_{2}}\left(\gamma_{2}\right), \ldots, F_{m_{L}}\left(\gamma_{L}\right)$, has the highest value. Then, a packet is transmitted over the specific hop having the highest CDF ordinate value using a TS. Naturally, due to the storeand-wait characteristics of the RNs, the potential performance improvement is obtained at the cost of an increased delay. In this contribution, we study both the block delay and the packet delay. The block delay is defined as the time required for a block of packets generated by the $\mathrm{SN}$ to reach the DN. By contrast, the packet delay is the time required for delivering a specific packet from the $\mathrm{SN}$ to the $\mathrm{DN}$, when assuming that there is an infinite number of packets to transmit. Furthermore, we also found that for a sufficiently large amount of data, the MHD scheme on average does not have to strike an explicit trade-off between the delay tolerated and the achievable error/outage performance. Our studies show that both our MHD scheme and the conventional multi-hop transmission scheme [19] use exactly the same number of TSs to deliver a block of packets, hence yielding the same block delay. This is because our MHD scheme transmits a single packet over one hop per TS, identically to the conventional multi-hop transmission scheme. By contrast, the packet delay is a random variable, distributed in a range bounded by the minimum and maximum packet delays. However, for an $L$ hop link, the MHD scheme delivers on average one packet per $L$ TSs to the DN, while the conventional multi-hop scheme delivers exactly one packet per $L$ TSs to the DN.

As mentioned in Section I, we have proposed a MHD scheme in $[16,17]$, which selects the specific hop having the highest SNR for transmitting a packet within a TS, which effectively implements selection diversity. In the MHD scheme proposed in $[16,17]$, we assumed that all the hops have the same average SNR and their channels experience the same fading, implying that the SNRs of all the hops obey the same $\mathrm{CDF}$. Hence, the selection procedure directly based on the SNRs of the $L$ hops would give each hop the same probability of $1 / L$ to be chosen. By contrast, in this paper, the SNRs of the $L$ hops obey different distributions, which depend on the $L$ parameters $m_{l}$, for $l=1,2, \ldots, L$. In this case, directly selecting the hop having the highest SNR would result in different activation probabilities for the $L$ links. Again, this is undesirable, since the hops chosen with relatively low probabilities may impose significant delays on the packet delivery, potentially resulting in a low throughput and possible bufferoverflow. Therefore, our proposed MHD scheme activates the specific hop, whose SNR maps to the maximum CDF ordinate value from the set $F_{m_{1}}\left(\gamma_{1}\right), F_{m_{2}}\left(\gamma_{2}\right), \ldots, F_{m_{L}}\left(\gamma_{L}\right)$, so that each hop has the same probability of $1 / L$ to be chosen for transmission within a TS.

Given the set of independent CDFs $F_{m_{1}}\left(\gamma_{1}\right)$, $F_{m_{2}}\left(\gamma_{2}\right), \ldots, F_{m_{L}}\left(\gamma_{L}\right)$ corresponding to $L$ hops, the proof that any hop is selected with a probability of $1 / L$ is as 
follows. Let us define a new random variable $X_{j}=F_{m_{j}}\left(\gamma_{j}\right)$ as the CDF of $\gamma_{j}$ which constitutes our selection criterion. It may be readily shown that $X_{j}$ are i.i.d for the different hops. In fact, the CDF of $X_{j}$ is expressed by ${ }^{2}$

$$
\begin{aligned}
F_{X_{j}}(x) & =P\left(X_{j}<=x\right) \\
& =P\left(F_{m_{j}}\left(\gamma_{j}\right)<=x\right) \\
& =P\left(\gamma_{j}<=F_{m_{j}}^{-1}(x)\right)
\end{aligned}
$$

(since the $\mathrm{CDF}$ is always an increasing function)

$$
\begin{aligned}
& =F_{m_{j}}\left(F_{m_{j}}^{-1}(x)\right) \text { (by definition) } \\
& =x \text { independent of } j,
\end{aligned}
$$

where $F^{-1}(\cdot)$ denotes the inverse function of the $\operatorname{CDF} F(\cdot)$. Therefore, $X_{j}$ are i.i.d (we already know that they are independent). Hence, we can say that the probability of selecting any hop is $\frac{1}{L}$.

In addition to the above property, this MHD scheme also has the following pair of desirable properties. Firstly, when the SNRs of all hops have the same CDF, selecting the hop with the maximum SNR from the set $\gamma_{1}, \gamma_{2}, \ldots, \gamma_{L}$ is equivalent to selecting the hop with the maximum CDF value, since this maximum CDF value is generated by the maximum SNR in the set of $\gamma_{1}, \gamma_{2}, \ldots, \gamma_{L}$. Secondly, our forthcoming discourse will demonstrate that similarly to the MHD scheme of [16, 17] the proposed MHD scheme is also capable of achieving full diversity. Based on the above properties, the MHD scheme proposed in this contribution may be viewed as a generalized MHD scheme, which achieves full multihop diversity, while guaranteeing that all hops have the same probability to be activated for transmission.

Note additionally that in [16] a decentralized MAC layer protocol was proposed for the implementation of the MHD scheme. There are three stages in this protocol. In the first two stages, each node may assume the knowledge of the buffer - fullness in its immediately adjacent nodes as well as the channel condition knowledge of nearby nodes within one or two hops. In the third stage, each node will broadcast different MAC layer control signals at specific time instant based on above-mentioned buffer and channel knowledge, as well as by exploiting the knowledge of the hop index of this node and of the previously received MAC layer control signal. Given this knowledge, the 'best' hop can be selected within no more than $\left(9+L \times S_{i}\right)$ symbol durations, where $L$ is the total number of hops and $S_{i}$ is the channel quality identifier. Physically this delays corresponds to the maximum MAC layer delay. Note that the delay of the first transmission is significantly lower than the maximum MAC layer delay. One of the specific examples given in [16] shows that the MAC layer delay is only $3.7 \%$ of the time slot duration. This MAC layer protocol can be directly used for the MHD scheme proposed in this contribution, after replacing $\gamma_{1}, \gamma_{2}, \ldots, \gamma_{L}$ of [16] by $F_{m_{1}}\left(\gamma_{1}\right), F_{m_{2}}\left(\gamma_{2}\right), \ldots, F_{m_{L}}\left(\gamma_{L}\right)$.

Having described the system model and the MHD scheme, below we focus our attention on the MHL's performance, including its BER, outage probability as well as diversity order.

\footnotetext{
${ }^{2}$ The authors gratefully acknowledge that this proof was generously provided by one of the anonymous reviewers.
}

The main assumptions adopted in our study are summarized as follows:

- The SN always has packets to send, hence the MHL operates in its steady state.

- The DN $D$ can store an infinite number of packets, while the RNs can only store at most $B$ packets.

- The fading processes of the $L$ hops of a MHL are independent, while the fading of a given hop remains constant within a packet duration, but it is independently faded from one packet to another.

\section{PERformance AnAlysis}

In this section, we first derive the lower-bound for the BER of the MHLs conveying BPSK/MQAM baseband signals. Then, the BER for the MHLs relying on the RNs having a buffer size $B$ is analyzed. Similarly, the lower-bound and exact outage probabilities are analyzed in this section. Due to the complexity to evaluate the exact BER and outage probability, when $L$ and $B$ are large, an approximation approach is proposed. Finally, the diversity order of our MHD scheme is derived.

Based on Fig. 1 and the operational principles of the MHLs described in Section II, the following events may occur, when every RN has a buffer size of $B$ packets. Firstly, the buffer of a RN may be empty at some instants. In this case, this RN cannot be the transmit node, since it has no packets to transmit. Secondly, the buffer of a RN may be full. Then, this RN cannot act as the receiver node, since its buffer is unable to store further packets. In both of the above cases, a hop has to be chosen for transmission from the resultant reduced set of hops. This results in an increased BER and outage probability due to the associated reduced selection diversity gain, as explained in our forthcoming discourse. Therefore, if we eliminate the above-mentioned constraints by assuming that every RN has an infinite buffer size, and that all the RNs can always transmit and receive packets, we can obtain the lower-bounds for both the BER and the outage probability of the MHLs. By contrast, when the exact performance analysis is considered, the effects of the finite buffer size have to be considered. In this case, we can carry out the relevant analysis based on the classic Markov chains [3]. Let us first analyze the achievable BER.

\section{A. Bit Error Rate Analysis}

In this section, the lower-bound single-hop BER for the lth hop, expressed as $P_{L e}^{(l)}$, is analyzed first, when every $\mathrm{RN}$ is assumed to have an infinite buffer for storing the received packets and also always have packets prepared for transmission. Then, the end-to-end lower-bound BER, $P_{L E}$, is derived, where the subscript ' $L E$ ' stands for 'lower-bound error rate'. Finally, the accurate single-hop and end-to-end BER are derived, when assuming that the RNs have buffers of finite size.

Based on the operational principles of the MHLs and the identical selection probability of every hop, when the $l$ th hop 


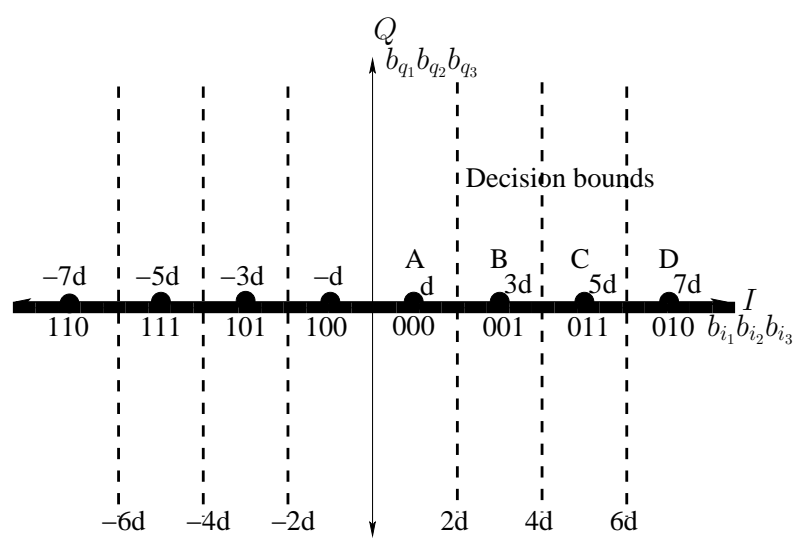

Fig. 2. IQ-axis mapping of the 64QAM constellation.

is activated, the instantaneous SNR of the selected hop obeys

$$
\begin{aligned}
& f_{l}^{S C}(\gamma) \\
= & \frac{L}{\left[\Gamma\left(m_{l}\right)\right]^{L}}\left(\frac{m_{l}}{\bar{\gamma}_{l}}\right)^{m_{l}} \gamma^{m_{l}-1}\left[\gamma\left(m_{l}, \frac{m_{l} \gamma}{\bar{\gamma}_{l}}\right)\right]^{L-1} \exp \left(-\frac{m_{l} \gamma}{\bar{\gamma}_{l}}\right), \\
& 0 \leq \gamma<\infty ; l=1,2, \ldots, L .
\end{aligned}
$$

It is interesting to observe from (7) that if the $l$ th hop is selected based on our MHD scheme, the PDF of its SNR is independent of the other $(L-1)$ channels. This conditional PDF is identical to that encountered, when the $L$ hops experience the same fading CDF as the lth hop. Furthermore, we can see that (7) is in fact the PDF of the maximum SNR selected from the set of SNRs $\left\{\gamma_{1}, \gamma_{2}, \ldots, \gamma_{L}\right\}$, when these SNRs obey independent identical distribution (i.i.d) with the $\mathrm{PDF}$ and $\mathrm{CDF}$ as given by (3). Hence, our MHD scheme effectively emulates a selection diversity scheme. The more hops are invoked, the higher the diversity gain becomes and therefore the better the BER performance becomes. Hence, when all the $L$ hops are assumed to be always available for activation, the BER obtained represents the lower-bound of the BER, which justifies the terminology of, lower-bound singlehop BER, and, lower-bound end-to-end BER, used in this paper.

Given the PDF $f_{l}^{S C}(\gamma)$, as shown in (7), the lower-bound single-hop BER for the $l$ th hop $P_{L e}^{(l)}$ can be derived by first considering the conditional probability $P_{L e}^{(l)}(\gamma)$. It is wellknown that the (square) MQAM signal can be decomposed into two independent PAM signals $[25,27,28]$, each of which has the constellation points located at

$$
\{ \pm d, \pm 3 d, \cdots, \pm(\sqrt{M}-1) d\}
$$

where $2 d$ represents the minimum Euclidean distance of the constellation points. When normalized by the noise's standard deviation $\sigma$, we have $[25,27]$

$$
\frac{d}{\sigma}=\sqrt{\frac{6 \gamma}{2(M-1)}}
$$

In MQAM, the two component PAM signals have the same error probability and can be treated independently. For example, when the classic Gray coded bit mapping is applied, which is the case considered in this paper, the 64QAM constellation can be decomposed into (I-)PAM and (Q-)PAM, as shown in Fig. 2, where $b_{i 1} b_{i 2} b_{i 3}$ and $b_{q 1} b_{q 2} b_{q 3}$ are the bits conveyed by the I-PAM and Q-PAM, respectively.

Let us specifically consider the I-PAM stream and express the probability $P_{i}$ that a transmitted signal belongs to the constellation point $i d$, where $i= \pm 1, \ldots, \pm(\sqrt{M}-1)$. Let $P_{\{i, j\}}^{(l)}(\gamma)$ represent the transition probability at the $l$ th hop, which is the probability that the receiver declares that $j d$ is detected, while $i d$ was transmitted. Furthermore, let $e_{i, j}$ be the number of different bits between the signal representing the constellation point $i d$ and that corresponding to the constellation point $j d$. Then, the BER of MQAM can be expressed as

$$
P_{L e}^{(l)}(\gamma)=\frac{2}{\log _{2} M} \sum_{i=-\sqrt{M}+1}^{\sqrt{M}-1} P_{i}\left(\sum_{j=-\sqrt{M}+1}^{\sqrt{M}-1} e_{i, j} P_{\{i, j\}}^{(l)}(\gamma)\right) .
$$

Let us define

$$
\begin{aligned}
\boldsymbol{p} & =\left[P_{-\sqrt{M}+1}, P_{-\sqrt{M}+3}, \cdots, P_{\sqrt{M}-1}\right]^{T} \\
\boldsymbol{P}_{l}(\gamma) & =\left[P_{\{i, j\}}^{(l)}(\gamma)\right], \boldsymbol{E}=\left[e_{i, j}\right], \mathbf{1}=[1,1, \cdots, 1]^{T},
\end{aligned}
$$

where $\boldsymbol{p}$ is an $\sqrt{M}$-length vector, 1 is an $\sqrt{M}$-length vector with elements of one, while $\boldsymbol{P}_{l}(\gamma)$ and $\boldsymbol{E}$ are $(\sqrt{M} \times \sqrt{M})$ dimensional square matrices. Then, (10) can be expressed in a compact form as

$$
\begin{aligned}
P_{L e}^{(l)}(\gamma) & =\frac{2}{\log _{2} M} \boldsymbol{p}^{T}\left[\boldsymbol{E} \odot \boldsymbol{P}_{l}(\gamma)\right] \mathbf{1} \\
& =\frac{2}{\log _{2} M} \mathbf{1}^{T}\left[\boldsymbol{E}^{T} \odot \boldsymbol{P}_{l}^{T}(\gamma)\right] \boldsymbol{p},
\end{aligned}
$$

where $\odot$ represents the Hadamard product [29]. Observe that at the right-hand side of (12), only $\boldsymbol{P}_{l}(\gamma)$ is a function of $\gamma$. Hence, the average single-hop BER $P_{L e}^{(l)}$ may be obtained by averaging $P_{L e}^{(l)}(\gamma)$ of (12) with respect to the PDF of (7), which can be expressed as

$$
\begin{aligned}
P_{L e}^{(l)} & =\int_{0}^{\infty} P_{L e}^{(l)}(\gamma) f_{l}(\gamma) d \gamma \\
& =\frac{2}{\log _{2} M} \mathbf{1}^{T}\left(\boldsymbol{E}^{T} \odot \int_{0}^{\infty} \boldsymbol{P}_{l}^{T}(\gamma) f_{l}^{S C}(\gamma) d \gamma\right) \boldsymbol{p} \\
& =\frac{2}{\log _{2} M} \mathbf{1}^{T}\left(\boldsymbol{E}^{T} \odot \boldsymbol{P}_{l}^{T}\right) \boldsymbol{p}
\end{aligned}
$$

where $\boldsymbol{P}_{l}=\left[P_{\{i, j\}}^{(l)}\right]$ with $P_{\{i, j\}}^{(l)}$ denoting the average transition probability from the constellation point $i$ to $j$, given by

$$
\begin{gathered}
P_{\{i, j\}}^{(l)}=\int_{0}^{\infty} P_{\{i, j\}}^{(l)}(\gamma) f_{l}^{S C}(\gamma) d \gamma \\
i, j= \pm 1, \pm 3, \ldots, \pm(\sqrt{M}-1)
\end{gathered}
$$

Again, $P_{\{i, j\}}^{(l)}(\gamma)$ is the transition probability that the receiver declares the $j$ th constellation point, given that the $i$ th 
constellation point was transmitted. This probability can be readily derived by referring to Fig. 2, which is

$P_{\{i, j\}}^{(l)}(\gamma)=\left\{\begin{array}{l}Q\left[(|i-j|-1) \frac{d}{\sigma}\right], \quad \text { when } j= \pm(\sqrt{M}-1) \\ Q\left[(|i-j|-1) \frac{d}{\sigma}\right]-Q\left[(|i-j|+1) \frac{d}{\sigma}\right], \text { others }\end{array}\right.$

where $Q(x)$ is the Gaussian $Q$-function. For example, when 4QAM (QPSK) is employed, we have $d / \sigma=\sqrt{\gamma}$. Hence, the probability transition matrix is expressed with the aid of (15) as

$$
\boldsymbol{P}_{l}(\gamma)=\left[\begin{array}{cc}
1-Q(\sqrt{\gamma}) & Q(\sqrt{\gamma}) \\
Q(\sqrt{\gamma}) & 1-Q(\sqrt{\gamma})
\end{array}\right]
$$

Finally, when substituting (7), (9) as well as (15) into (14) and completing the integration, $P_{i, j}$ can be expressed as

$P_{\{i, j\}}^{(l)}=\left\{\begin{array}{l}\bar{Q}_{l}\left(\sqrt{(|i-j|-1) \bar{\gamma}_{l}}\right), \quad \text { when } j= \pm(\sqrt{M}-1) \\ \bar{Q}_{l}\left(\sqrt{(|i-j|-1) \bar{\gamma}_{l}}\right)-\bar{Q}_{l}\left(\sqrt{(|i-j|+1) \bar{\gamma}_{l}}\right), \\ \text { others, }\end{array}\right.$

By letting $A_{i, j}=|i-j|-1$ or $A_{i, j}=|i-j|+1, \bar{Q}_{l}\left(\sqrt{A_{i, j} \bar{\gamma}_{l}}\right)$ in the above equation is given by

$$
\begin{aligned}
\bar{Q}_{l}\left(\sqrt{A_{i, j} \bar{\gamma}_{l}}\right)= & \left(\frac{m_{l}}{\bar{\gamma}_{l}}\right)^{m_{l}} \frac{L}{\left(\Gamma\left(m_{l}\right)\right)^{L}} \int_{0}^{\infty} Q\left(\sqrt{A_{i, j} \gamma}\right) \gamma^{m_{l}-1} \\
& \times\left[\gamma\left(m_{l}, \frac{m_{l} \gamma}{\bar{\gamma}_{l}}\right)\right]^{L-1} \exp \left(-\frac{m_{l} \gamma}{\bar{\gamma}_{l}}\right) d \gamma
\end{aligned}
$$

When $m_{l}$ is an arbitrary non-integer value, we find that it is extremely hard to derive the closed-form solution for the integral in (18). By contrast, when $m$ is an integer, a closedform expression can be derived for (18). In this case, firstly, the incomplete Gamma function can be expressed as [21](8.352.6)

$$
\frac{\gamma(m, x)}{\Gamma(m)}=\left(1-e^{-x} \sum_{n=0}^{m-1} \frac{x^{n}}{n !}\right) .
$$

Then, upon substituting (19) into (18) and after some rearrangement, we arrive at

$$
\begin{aligned}
& \bar{Q}_{l}\left(\sqrt{A_{i, j} \bar{\gamma}_{l}}\right) \\
= & \left(\frac{m_{l}}{\bar{\gamma}_{l}}\right)^{m_{l}} \frac{L}{\Gamma\left(m_{l}\right)} \sum_{l_{i}=0}^{L-1}(-1)^{l_{i}}\left(\begin{array}{c}
L-1 \\
l_{i}
\end{array}\right) \sum_{k=0}^{l_{i}\left(m_{l}-1\right)} c_{k}^{\left(l_{i}\right)}\left(\frac{m_{l}}{\bar{\gamma}_{l}}\right)^{k} \\
& \times \int_{0}^{\infty} Q\left(\sqrt{A_{i, j} \gamma}\right) \gamma^{k+m_{l}-1} \exp \left(-\frac{m_{l}\left(l_{i}+1\right)}{\bar{\gamma}_{l}} \gamma\right) d \gamma .
\end{aligned}
$$

Finally, the above expression can be simplified with the aid of the results from $[26,30]$,

$$
\begin{aligned}
& \bar{Q}_{l}\left(\sqrt{A_{i, j} \bar{\gamma}_{l}}\right) \\
= & \frac{L}{\Gamma\left(m_{l}\right)} \sum_{l_{i}=0}^{L-1}(-1)^{l_{i}}\left(\begin{array}{c}
L-1 \\
l_{i}
\end{array}\right) \sum_{k=0}^{l_{i}\left(m_{l}-1\right)} c_{k}^{\left(l_{i}\right)} \frac{\Gamma\left(k+m_{l}\right)}{\left(l_{i}+1\right)^{m_{l}+k}} \\
& \times \frac{1}{2}\left[1-\mu \sum_{h=0}^{m_{l}+k-1}\left(\begin{array}{c}
2 h \\
h
\end{array}\right)\left(\frac{1-\mu^{2}}{4}\right)^{h}\right]
\end{aligned}
$$

where the coefficients $c_{k}^{\left(l_{i}\right)}$ can be recursively computed according to the formulas in (16) of [26], yielding

$$
\begin{gathered}
c_{0}^{\left(l_{i}\right)}=1, c_{1}^{\left(l_{i}\right)}=l_{i}, c_{l_{i}\left(m_{l}-1\right)}^{\left(l_{i}\right)}=\frac{1}{\left[\left(m_{l}-1\right) !\right]^{l_{i}}} \\
c_{k}^{\left(l_{i}\right)}=\frac{1}{k} \sum_{j=1}^{\min \left(k, m_{l}-1\right)} \frac{j\left(l_{i}+1\right)-k}{j !} c_{k-j}^{\left(l_{i}\right)} .
\end{gathered}
$$

Furthermore, in (21), we have for $M \geq 4$

$$
\mu=\sqrt{\frac{A_{i, j}^{2} g \bar{\gamma}_{l}}{2 m_{l}\left(l_{i}+1\right) / \bar{\gamma}_{l}+A_{i, j}^{2} g \bar{\gamma}_{l}}}, \text { with } g=\frac{1.5}{M-1},
$$

and, for BPSK $(M=2)$, we have $A_{i, j}=2, g=0.5$ and, correspondingly, $\mu=\sqrt{\frac{\bar{\gamma}_{l}}{m_{l}\left(l_{i}+1\right) / \gamma_{h}+\bar{\gamma}_{l}}}$ and $P_{L e}^{(l)}=\bar{Q}_{l}\left(\sqrt{2 \bar{\gamma}_{l}}\right)$.

Finally, we note that the lower-bound single-hop BER $P_{L e}^{(l)}$ of the $L$-hop MHL supported by the proposed MHD principles can be evaluated by substituting (17) and the associated formulas into (13).

Having obtained the lower-bound single-hop BER $P_{L e}^{(l)}$ of (13), the lower-bound end-to-end BER $P_{L E}$ can now be derived by exploiting that the decode-and-forward scheme of Fig. 1, where a packet is passed through the entire ad hoc chain from one node to another. Regardless of the number of hops, we are interested in the end-to-end constellation-constellation transition matrix. ${ }^{3}$ Once we determined this transition matrix, the BER performance can be analyzed similarly to the single hop case. This equivalent end-to-end constellationconstellation transition matrix is $\prod_{l=1}^{L} \boldsymbol{P}_{l}^{T}$, which is created as the product of the transition matrix in each hop of the link. Hence, when considering all the $\sqrt{M}$ possible transmitted symbols, which have the a-priori probabilities of $\boldsymbol{p}$, as shown in (11), the lower-bound end-to-end average BER of the $L$-hop MHL can be expressed as (12), yielding

$$
P_{L E}=\frac{2}{m} \mathbf{1}^{T}\left[\boldsymbol{E}^{T} \odot\left[\prod_{l=1}^{L} \boldsymbol{P}_{l}^{T}\right]\right] \boldsymbol{p} .
$$

Note that, an end-to-end average BER expression for MHLs has been derived in [31](42), which has a similar form as (24). However, the expression [31](42) is only for BPSK/QPSK, but not for MQAM, when $M \geq 16$. Specifically, for BPSK we can readily obtain the lower-bound end-to-end BER expression, which is

$$
P_{L E}=\frac{1}{2}-\frac{1}{2} \prod_{l=1}^{L}\left(1-2 P_{L e}^{(l)}\right) .
$$

The lower-bound end-to-end BER of the MHLs has been derived above, which assumed that each $\mathrm{RN}$ has an infinite buffer size and the RNs always have packets to transmit. The exact end-to-end BER of the MHLs is considered below, where we assumed that every RN has a buffer of finite size. In this case, the hops obeying either of the following conditions should be excluded from the transmission list: a) the hops, whose transmit nodes do not have packets to transmit

\footnotetext{
${ }^{3}$ The constellation-constellation transition matrix represents the transition probabilities from constellation points from transmitter to constellations points at the receiver.
} 
and b) the hops, whose receive nodes are unable to accept packets, since their buffers are full. When taking the above two situations into account, the exact end-to-end BER of MHLs can be analyzed as follows.

When deriving the accurate BER of the MHD scheme, we have to consider the constraint that the $L$-hop link is forced to choose the best one from the set of $\hat{l}$ hops in order to send its information, when $(L-\hat{l})$ out of the $L$ hops are unable to transmit information. This happens either when some of the transmit nodes' buffers are empty or when some of the receive nodes' buffers are full. Before finding the exact endto-end BER $P_{e}$ with finite size of buffer, the concept of state is proposed. Based on this concept, the packets transmitted over an $L$-hop link under the MHD scheme can be described as a Markov process.

Let us assume that the buffer size of every $\mathrm{RN}$ is $B$ packets. When MQAM modulation is employed, then $\log _{2} M$ packets are transmitted simultaneously. Hence, we can express the equivalent buffer size of a $\mathrm{RN}$ as $\hat{B}=B / \log _{2} M$ packets. Let the numbers of packets stored in the $(L-1)$ RNs, $R_{1}, R_{2}, \ldots, R_{L-1}$, be $b_{1}, b_{2}, \ldots, b_{L-1}$, where $b_{l}=$ $0,1, \ldots, B$. Then, upon considering MQAM, the corresponding equivalent numbers of packets are $\hat{b}_{1}, \hat{b}_{2}, \ldots, \hat{b}_{L-1}$, where $\hat{b}_{i}=b_{i} / \log _{2} M$. Given the above definitions, the states of the $L$-hop link can be defined in terms of the number of packets stored in the $(L-1)$ RNs as

$$
S_{i}=\left[\hat{b}_{1}^{(i)}, \hat{b}_{2}^{(i)}, \cdots, \hat{b}_{L-1}^{(i)}\right]^{T}, i=0,1, \ldots, N-1,
$$

where $\hat{b}_{l}^{(i)}$ denotes the equivalent number of packets stored in the $l$ th $\mathrm{RN}$, when the $L$-hop link is in state $i$, while $N=$ $(\hat{B}+1)^{L-1}$ is the total number of states of the Markov process, which constitute a set $\mathcal{S}=\left\{S_{0}, S_{1}, \ldots, S_{N-1}\right\}$. Based on the transitions among the $N$ states, a state transition matrix denoted by $T$ can be characterized by the state transition probabilities $\left\{P_{i j}=P\left(s(t+1)=S_{j} \mid s(t)=S_{i}\right), i, j=\right.$ $0,1, \ldots, N-1\}$.

For example, when considering a three-hop link having the parameters of $L=3$ and $\hat{B}=2$, there is a total of $N=3^{2}=9$ states, which form a set

$$
\begin{aligned}
\mathcal{S}=\left\{S_{0}\right. & =[0,0]^{T}, S_{1}=[0,1]^{T}, S_{2}=[0,2]^{T}, \\
S_{3} & =[1,0]^{T}, S_{4}=[1,1]^{T}, S_{5}=[1,2]^{T}, \\
S_{6} & \left.=[2,0]^{T}, S_{7}=[2,1]^{T}, S_{8}=[2,2]^{T}\right\} .
\end{aligned}
$$

Correspondingly, the state transition matrix becomes:

$$
\boldsymbol{T}=\left[\begin{array}{ccccccccc}
0 & 0 & 0 & 1 & 0 & 0 & 0 & 0 & 0 \\
1 / 2 & 0 & 0 & 0 & 1 / 2 & 0 & 0 & 0 & 0 \\
0 & 1 / 2 & 0 & 0 & 0 & 1 / 2 & 0 & 0 & 0 \\
0 & 1 / 2 & 0 & 0 & 0 & 0 & 1 / 2 & 0 & 0 \\
0 & 0 & 1 / 3 & 1 / 3 & 0 & 0 & 0 & 1 / 3 & 0 \\
0 & 0 & 0 & 0 & 1 / 2 & 0 & 0 & 0 & 1 / 2 \\
0 & 0 & 0 & 0 & 1 & 0 & 0 & 0 & 0 \\
0 & 0 & 0 & 0 & 0 & 1 / 2 & 1 / 2 & 0 & 0 \\
0 & 0 & 0 & 0 & 0 & 0 & 0 & 1 & 0
\end{array}\right] .
$$

Although the transition matrix $T$ may be large, it can be readily constructed. An algorithm conceived for forming the matrix $T$ may be described as follows:

1) The $(\hat{B}+1)^{L-1} \times(\hat{B}+1)^{L-1}$ matrix $T$ is first initialized with zero elements.

2) For row $i, i=0,1, \ldots,(\hat{B}+1)^{L-1}-1$, which corresponds to the $i$ th state $S_{i}=\left[\hat{b}_{1}^{(i)}, \hat{b}_{2}^{(i)}, \cdots, \hat{b}_{L-1}^{(i)}\right]^{T}$, the following operations are carried out:

- If $\hat{b}_{1}^{(i)}+1 \leq \hat{B}$, the column corresponding to the output state $\left[\hat{b}_{1}^{(i)}+1, \hat{b}_{2}^{(i)}, \cdots, \hat{b}_{L-1}^{(i)}\right]^{T}$ is set to one;

- For $l=1,2, \ldots, L-2$, if $b_{l}^{(i)}-1 \geq 0$ and $\hat{b}_{l+1}^{(i)}+1 \leq$ $\hat{B}$, the column corresponding to the output state $\left[\hat{b}_{1}^{(i)}, \ldots, \hat{b}_{l}^{(i)}-1, \hat{b}_{l+1}^{i}+1, \cdots, \hat{b}_{L-1}^{(i)}\right]^{T}$ is set to one;

- If $\hat{b}_{L-1}^{(i)}-1 \geq 0$, the column corresponding to the output state $\left[\hat{b}_{1}^{(i)}, \hat{b}_{2}^{(i)}, \cdots, \hat{b}_{L-1}^{(i)}-1\right]^{T}$ is set to one.

3) Each of the rows is divided by the number of ones in the same row, to guarantee that the total transition probability from state $S_{i}$ is one.

It can be shown that the state transition matrix $T$ has the following properties:

- Matrix $T$ is a sparse matrix. Every row has at most $L$ number of non-zero elements, while the remaining at least $(\hat{B}+1)^{L-1}-L$ number of elements are zero elements.

- The sum of the probabilities in each row is one;

- The number of non-zero elements in a row represents the number of hops that may be chosen for transmission.

Having obtained the state transition matrix $\boldsymbol{T}$, the steadystate probabilities can be computed by the formula [32]

$$
\pi=T^{T} \pi,
$$

where $\pi=\left[\pi_{0}, \pi_{1}, \ldots, \pi_{(\hat{B}+1)^{L-1}-1}\right]^{T}$, in which $\pi_{i}$ (or $\pi_{S_{i}}$ ) is the steady-state probability that the $L$-hop link is in state $S_{i}$. Note that $\pi$ in (28) is the right eigenvector of the matrix $T^{T}$ associated with an eigenvalue of one. Therefore, $\pi$ can be obtained by solving the corresponding eigenvector problem [33]. However, the matrix $\boldsymbol{T}$ may be extreme by large. An approximate method of generating $\pi$ will be provided in Subsection III-B.

The next step is to find the exact end-to-end BER $P_{e}$ based on $\boldsymbol{\pi}$. Firstly, a $(L \times L)$ element matrix $\boldsymbol{P}_{\text {sel }}^{\hat{B}}$ is computed, in which $\boldsymbol{P}_{\text {sel }}^{\hat{B}}(l, \hat{l})$ denotes the probability that the $l$ th hop is selected from $\hat{l}$ available hops, when the equivalent buffer size per RN is $\hat{B}$. We assume that the total number of available hops corresponding to state $S_{i}$ is $N_{S_{i}}$. Then, $\boldsymbol{P}_{\text {sel }}^{\hat{B}}$ can be determined by the following algorithm.

Algorithm 1:

1) Initialization: $\boldsymbol{P}_{\text {sel }}^{\hat{B}}=\mathbf{0}$;

2) for $i=1:(\hat{B}+1)^{L-1} / /$ all the $(\hat{B}+1)^{L-1}$ possible states;

for $j=1: N_{S_{i}} / /$ all the available hops in state $S_{i}$; compute:

$$
\boldsymbol{P}_{\text {sel }}^{\hat{B}}\left(j_{S_{i}}, N_{S_{i}}\right)=\boldsymbol{P}_{\text {sel }}^{\hat{B}}\left(j_{S_{i}}, N_{S_{i}}\right)+\frac{\pi_{i}}{N_{S_{i}}} ;
$$


In (29), $j_{S_{i}} \in\{1,2, \ldots, L\}$ denotes the hop index of the $j$ th available hop in state $S_{i}$. After obtaining $\boldsymbol{P}_{\text {sel }}^{\hat{B}}$ of (29), the average BER $\boldsymbol{p}_{l}^{\hat{B}}$ of the $l$ th hop can be computed with the aid of the whole probability formula, yielding

$$
\boldsymbol{p}_{l}^{\hat{B}}=L \sum_{\hat{l}=1}^{L} \boldsymbol{P}_{\text {sel }}^{\hat{B}}(l, \hat{l}) P_{L e}^{(\hat{l})},
$$

where $P_{L e}^{(\hat{l})}$ is given by (13). Finally, the exact end-to-end BER of the MHL of the RNs can be obtained for a finite buffer by substituting (30) into (24).

\section{B. Approximate solution of (28)}

The accurate value of $\pi$ can be obtained by solving (28), when $T$ is small. By contrast, if $T$ is large, the achievable performance approaches the theoretical bound, which will be detailed in Section IV. If the size of $T$ is mediocre, an approximate method is provided here for circumventing this problem. Note that all our discussions are based on assuming steady-state operation.

Actually, the number of packets in the RN buffers cannot be considered independent. Nevertheless, for the sake of simplicity, they are treated as independent for the sake of having the relevant approximate expressions. Assuming that $p_{i, \hat{b}}$ represents the probability of having $\hat{b}$ packets in the $i$ th relay, we can approximate $\pi_{S_{j}=\left[\hat{b}_{1}^{(j)}, \hat{b}_{2}^{(j)}, \ldots, \hat{b}_{L-1}^{(j)}\right]^{T}} \approx \prod_{i=1}^{L-1} p_{i, \hat{b}_{i}^{(j)}}$. Based on this formula, we can see that in order to compute $\pi_{S_{j}=\left\{\hat{b}_{1}^{(j)}, \hat{b}_{2}^{(j)}, \ldots, \hat{b}_{L-1}^{(j)}\right]^{T}}$ for all the $(\hat{B}+1)^{L-1}$ states, the total number of unknown probabilities is $(L-1)(\hat{B}+1)$, which are $p_{i, \hat{b}}$ for $i=1,2, \ldots, L-1$ and $b=0,1, \ldots, \hat{B}$. Hence, our objective is to express these probabilities by finding at least $(L-1)(\hat{B}+1)$ equations, which are derived below by exploiting their specific properties.

1) Symmetry Proposition: Let the probability of a state $S_{i}=\left[\hat{b}_{1}^{(i)}, \hat{b}_{2}^{(i)}, \cdots, \hat{b}_{L-1}^{(i)}\right]^{T}, i=0,1, \ldots, N-$ 1 be $\pi_{i}$. Let the probability of another state $S_{\bar{i}}=$ $\left[\hat{B}-\hat{b}_{L-1}^{(i)}, \hat{B}-\hat{b}_{L-2}^{(i)}, \cdots, \hat{B}-\hat{b}_{1}^{(i)}\right]^{T}$ be $\pi_{\bar{i}}$, where $\hat{b}_{l}^{(i)}$ for $l=1, \ldots, L-1$ are given by the corresponding $\hat{b}_{l}^{(i)}$ in $S_{i}$. Then we have $\pi_{\bar{i}}=\pi_{i}$.

Proof: The unavailable channels are those ones that are linked to the specific RNs having either an empty or a full buffer. The channels available for state $S_{i}$ are the same as for state $S_{\bar{i}}$. Since every node that has zero packets to send in $S_{i}$ is treated as a node having $\hat{B}$ packets, this represents a full buffer. Hence it will be unable to receive any packets. By contrast, the nodes that have $\hat{B}$ packets to send in $S_{i}$ are treated as nodes having zero packets. Since this is a node with an empty buffer, it will be unable to transmit any packet. Therefore, the number of available channels remains unchanged. The following is the mathematical proof. If the state $S_{i}$ is substituted by $S_{\bar{i}}$ in (28), the equation remains unchanged. Therefore, we have $\pi_{\bar{i}}=\pi_{i}$.

Let us consider the same example as before. Again, the relevant parameters are $L=3$ and $\hat{B}=2$. Then, from (28)

$$
\begin{array}{r}
\pi_{S_{0}=[0,0]^{T}}=\pi_{S_{1}=[0,1]^{T}} \times \frac{1}{2} \\
(31) \\
\pi_{S_{1}=[0,1]^{T}}=\pi_{S_{2}=[0,2]^{T}} \times \frac{1}{2}+\pi_{S_{3}=[1,0]^{T}} \times \frac{1}{2} \\
\pi_{S_{2}=[0,2]^{T}}=\pi_{S_{4}=[1,1]^{T}} \times \frac{1}{3} \\
\pi_{S_{3}=[1,0]^{T}}=\pi_{S_{0}=[0,0]^{T}} \times 1+\pi_{S_{4}=[1,1]^{T}} \times \frac{1}{3} \\
\pi_{S_{5}=[1,2]^{T}}=\pi_{S_{2}=[0,2]^{T}} \times \frac{1}{2}+\pi_{S_{7}=[2,1]^{T}} \times \frac{1}{2} \\
\pi_{S_{6}=[2,0]^{T}}=\pi_{S_{3}=[1,0]^{T}} \times \frac{1}{2}+\pi_{S_{7}=[2,1]^{T}} \times \frac{1}{2} \\
\pi_{S_{7}=[2,1]^{T}}=\pi_{S_{4}=[1,1]^{T}} \times \frac{1}{3}+\pi_{S_{8}=[2,2]^{T}} \times \frac{1}{2}+\pi_{S_{5}=[1,2]^{T}} \times \frac{1}{2}+\pi_{S_{6}=[2,0]^{T}} \times \frac{1}{2} \\
\pi_{S_{8}=[2,2]^{T}}=\pi_{S_{5}=[1,2]^{T}} \times \frac{1}{2} .
\end{array}
$$

If all $S_{i}$ values are substituted by $S_{\bar{i}}$, the above formula becomes:

$$
\begin{aligned}
& \pi_{S_{8}=[2,2]^{T}}=\pi_{S_{5}=[1,2]^{T}} \times \frac{1}{2} \\
& \pi_{S_{5}=[1,2]^{T}}=\pi_{S_{2}=[0,2]^{T}} \times \frac{1}{2}+\pi_{S_{7}=[2,1]^{T}} \times \frac{1}{2} \\
& \pi_{S_{2}=[0,2]^{T}}=\pi_{S_{4}=[1,1]^{T}} \times \frac{1}{3} \\
& \pi_{S_{7}=[2,1]^{T}}=\pi_{S_{8}=[2,2]^{T}} \times 1+\pi_{S_{4}=[1,1]^{T}} \times \frac{1}{3} \\
& \pi_{S_{4}=[1,1]^{T}}=\pi_{S_{5}=[1,2]^{T}} \times \frac{1}{2}+\pi_{S_{1}=[0,1]^{T}} \times \frac{1}{2}+\pi_{S_{6}=[2,0]^{T}} \times 1 \\
& \pi_{S_{1}=[0,1]^{T}}=\pi_{S_{2}=[0,2]^{T}} \times \frac{1}{2}+\pi_{S_{3}=[1,0]^{T}} \times \frac{1}{2} \\
& \pi_{S_{6}=[2,0]^{T}}=\pi_{S_{7}=[2,1]^{T}} \times \frac{1}{2}+\pi_{S_{3}=[1,0]^{T}} \times \frac{1}{2} \\
& \pi_{S_{3}=[2,1]^{T}}=\pi_{S_{4}=[1,1]^{T}} \times \frac{1}{3}+\pi_{S_{0}=[0,0]^{T}} \times 1 \\
& \pi_{S_{0}=[2,2]^{T}}=\pi_{S_{1}=[0,1]^{T}} \times \frac{1}{2},
\end{aligned}
$$

which is identical to (31). The only difference is in the terminology. Hence, the solution of (32) is also the same as that of (31), which means that $\pi_{S_{0}}=\pi_{S_{8}}, \pi_{S_{1}}=\pi_{S_{5}}$, $\pi_{S_{3}}=\pi_{S_{7}}$, as so on. 
Based on the proposition above, we arrive at

$$
\begin{aligned}
& p_{i, \hat{b}} \\
= & \sum_{j_{1}=0}^{\hat{B}} \sum_{j_{2}=0}^{\hat{B}} \cdots \sum_{j_{i-1}=0}^{\hat{B}} \sum_{j_{i+1}=0}^{\hat{B}} \cdots \sum_{i_{L-1}=0}^{\hat{B}} \\
& \times \pi_{S=\left[j_{1}, j_{2} \cdots, j_{i-1}, \hat{b}, j_{i+1} \cdots, j_{L-1}\right]^{T}} \\
= & \sum_{j_{1}=0}^{\hat{B}} \sum_{j_{2}=0}^{\hat{B}} \cdots \sum_{j_{i-1}=0}^{\hat{B}} \sum_{j_{i+1}=0}^{\hat{B}} \cdots \sum_{i_{L-1}=0}^{\hat{B}} \\
& \times \pi_{S=\left[\hat{B}-j_{L-1} \cdots, \hat{B}-j_{i+1}, \hat{B}-\hat{b}, \hat{B}-j_{i-1}, \cdots, \hat{B}-j_{2}, \hat{B}-j_{1}\right]^{T}} \\
= & p_{L-i, \hat{B}-\hat{b}}, i=1,2, \ldots, L-1 ; \quad b=0,1, \ldots,\lfloor(\hat{B}+1) / 2\rfloor .
\end{aligned}
$$

where $\lfloor x\rfloor$ returns the maximal integer less than $x$. From (33), we can see that the proposition can provide $\lfloor(L-1)(\hat{B}+1) / 2\rfloor$ equations.

2) The probability of using every channel is identical: When our MHD scheme is employed and when the MHL is operated in steady state, every hop has approximately the same probability to be activated. According to the operational principles of the MHD, as described in Section II, if the $i$ th hop is selected, the $(i-1)$ st node's buffer must not be empty, which has a probability of $\left(1-p_{i-1,0}\right)$, and the $i$ th node's buffer should not be full, which has a probability of $\left(1-p_{i, \hat{B}}\right)$. In addition to the above mentioned buffer condition, let us assume that every hop has a $\frac{1}{L}$ probability of experiencing the "best" channel. Naturally, this is not always exactly satisfied, hence it remains an approximation. For the $\mathrm{SN}$ and DN, we set $p_{0,0}=0$ and $p_{L, \hat{B}}=0$, since we assumed that the SN always has packets to transmit and the $\mathrm{DN}$ is always ready to receive a packet. Hence, when considering all the $L$ hops, we have the following relationship

$$
\begin{aligned}
& \left(1-p_{0, \hat{B}}\right) \approx\left(1-p_{i-1,0}\right)\left(1-p_{i, \hat{B}}\right) \\
& \approx\left(1-p_{j-1,0}\right)\left(1-p_{j, \hat{B}}\right) \approx\left(1-p_{L, 0}\right), \\
& \quad i=1, \ldots, L-1 ; j=i+1, i+2, \ldots, L .
\end{aligned}
$$

From (34), we can construct a total of $L(L-1) / 2$ equations. However, some of them can be derived from some others, i.e. they are not independent. After a close consideration and applying the above "Symmetry Proposition", we arrive at $\lfloor(L-1) / 2\rfloor$ independent equations.

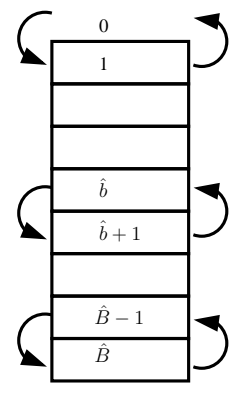

Fig. 3. Considering node $i$, the probability of preceding from $\hat{b}$ to $\hat{b}+1$ is the same as that from $\hat{b}+1$ to $\hat{b}$, when system is steady. If the buffer of node $i$ is empty, the $(i+1)$ th hop is unavailable, while if that of node $i$ is full, the $i$ th hop is unavailable.
3) The system is in its steady state: When this is in the case, for a single RN, the average number of transitions from $\hat{b}$ to $\hat{b}+1$ is the same as that from $\hat{b}+1$ to $\hat{b}$. By referring to Fig. 3, we may readily infer that for $\hat{b}=1,2, \ldots, \hat{B}-2$, we have

$$
\begin{array}{r}
p_{i, \hat{b}}\left(1-p_{i-1,0}\right) / L \approx p_{i, \hat{b}+1}\left(1-p_{i+1, \hat{B}}\right) / L, \\
i=1,2, \ldots, L-1,
\end{array}
$$

where $\left(1-p_{i-1,0}\right) / L$ at the left-hand side denotes the probability that node $i$ receives a packet from node $(i-1)$, while $\left(1-p_{i+1, \hat{B}}\right) / L$ is the probability that node $i$ sends a packet to node $(i+1)$.

When $\hat{b}=0$, the $(i+1)$ th hop is unavailable, as the $i$ th node's buffer is empty. Hence, the transitions satisfy

$$
\begin{array}{r}
p_{i, 0}\left(1-p_{i-1,0}\right) /(L-1) \approx p_{i, 1}\left(1-p_{i+1, \hat{B}}\right) / L \\
i=1,2, \ldots, L-1
\end{array}
$$

Similarly, when $\hat{b}=\hat{B}-1$, we have

$$
\begin{array}{r}
p_{i, \hat{B}-1}\left(1-p_{i-1,0}\right) / L \approx p_{i, \hat{B}}\left(1-p_{i+1, \hat{B}}\right) /(L-1), \\
i=1,2, \ldots, L-1 .
\end{array}
$$

where the factor $1 /(L-1)$ at the righthand side is because there are only $(L-1)$ hops avaliable for selection, when node $i$ is at state $\hat{B}$ (full), resulting in that the $i$ hop is unavailable.

Consequently, when considering (35) - (37) and "Symmetry Proposition", we can obtain $\hat{B}(L-1) / 2$ approximate equations.

4) The total probability is unity: Finally, the sum of the probabilities of $p_{i, \hat{b}}$ at $\mathrm{RN} i$ should satisfy:

$$
\sum_{\hat{b}=0}^{\hat{B}} p_{i, \hat{b}}=1, i=1,2, \ldots, L-1 .
$$

When considering the "Symmetry Proposition", we arrive at $\lfloor(L-1) / 2\rfloor$ equations.

By exploiting the properties of the MHL operated under our proposed MHD scheme, we can demonstrate that the number of equations is no less than the number of unknowns. If $L$ is odd and $\hat{B}>1$, the number of equations is given by

$$
\begin{aligned}
& \lfloor(L-1)(\hat{B}+1) / 2\rfloor+\underbrace{\lfloor L / 2\rfloor+\lfloor(L-1) / 2\rfloor}_{\mathrm{L}-1}+\hat{B}(L-1) / 2 \\
= & \frac{L-1}{2}(\hat{B}+1)+(L-1)+\hat{B} \frac{L-1}{2} \\
= & (L-1)\left(\hat{B}+\frac{3}{2}\right),
\end{aligned}
$$

which is larger than the number of unknowns $(L-1)(\hat{B}+1)$. By contrast, if $L$ is even and $\hat{B}>1$, the number of equations obeys:

$$
\begin{aligned}
& \lfloor(L-1)(\hat{B}+1) / 2\rfloor+\underbrace{\lfloor L / 2\rfloor+\lfloor(L-1) / 2\rfloor}_{\mathrm{L}-1}+\hat{B}(L-1) / 2 \\
= & \frac{L-1}{2}(\hat{B}+1)-\frac{1}{2}+(L-1)+(L-1)(\hat{B} / 2) \\
= & (L-1)\left(\hat{B}+\frac{3}{2}\right)-\frac{1}{2},
\end{aligned}
$$


which is larger than the number of unknowns $(L-1)(\hat{B}+1)$. All the above discussion is based on the assumption of $\hat{B}>1$. This is because the size of $\boldsymbol{T}$ is $2^{L-1} \times 2^{L-1}$ when $\hat{B}=1$, which is not large.

Hence, we can express the $(L-1)(\hat{B}+1)$ probabilities $p_{i, \hat{b}_{i}}$ for $i=1,2, \ldots, L-1$ and $\hat{b}_{i}=0,1, \ldots, \hat{B}$ by solving $(L-$ 1) $(\hat{B}+1)$ number of the equations. Consequently, the $(\hat{B}+$ $1)^{L-1}$ steady state probabilities $\left\{\pi_{S_{j}}\right\}$ can be approximately determined using the relationship of $\pi_{S_{j}=\left[\hat{b}_{1}^{(j)}, \hat{b}_{2}^{(j)}, \ldots, \hat{b}_{L-1}^{(j)}\right]^{T}} \approx$ $\prod_{i=1}^{L-1} p_{i, \hat{b}_{i}^{(j)}}$.

Specifically, a four step algorithm may be formulated as follows. Step one: Take all $p_{i, 0}$ as unknowns. Step two: Based on (33), all $p_{i, \hat{B}}$ can be determined. Step three: Based on (35), (36) and (37), all other $p_{i, \hat{b}}$ can be found. Step four: The $p_{i, 0}$ for $i=1,2, \ldots, L-1$ are solved based on (38). This can be achieved, since there are $(L-1)$ equations for $(L-1)$ unknowns.

Below we consider the special case of two hops. In this case, all the approximate formulas provided above become accurate, yielding:

$$
\begin{aligned}
& \pi_{0}=\pi_{\hat{B}}=\frac{1}{2 \hat{B}} \\
& \pi_{i}=\frac{1}{\hat{B}}, i=1,2, \cdots, \hat{B}-1,
\end{aligned}
$$

which can be obtained either from the equations provided in this subsection or directly from (28). Note that, in (28), each line has no more then two unknowns. We assume $\pi_{0}=\kappa$ and $\pi_{i}=2 \kappa, i=1,2, \cdots, \hat{B}-1$, as well as $\pi_{\hat{B}}=\kappa$. Finally, by exploiting (38) we arrive at $\kappa=\frac{1}{2 \hat{B}}$.

Let us now analyze the outage probability (OP).

\section{Outage Probability}

The OP characterizes the specific event that the instantaneous SNR of the selected hop is lower than a pre-set threshold. When an outage occurs, no data will be transmitted over the MHL, in order to avoid violating the required target BER, since otherwise the BER would become higher than the target. Therefore, given the set of thresholds $\gamma_{T_{l}}$ for the $L$ hops, the lower-bound OP is

$$
P_{L O}=\frac{1}{L} \sum_{l=1}^{L}\left[\int_{0}^{\gamma_{T_{l}}} f_{l}^{S C}(\gamma) d \gamma\right],
$$

when assuming that all the $L$ hops have the same probability of $1 / L$ being activated. Upon substituting $f_{l}^{S C}(\gamma)$ from (7) into the above equation, we readily arrive at:

$$
P_{L O}=\frac{1}{L} \sum_{l=1}^{L}\left[\frac{\gamma\left(m_{l}, \frac{m_{l} \gamma_{T_{l}}}{\bar{\gamma}_{l}}\right)}{\Gamma\left(m_{l}\right)}\right]^{L} .
$$

Furthermore, given $\boldsymbol{\pi}$ and $\boldsymbol{P}_{\text {sel }}^{\hat{B}}$, the exact OP of the MHLs whose RNs have a finite buffer can be expressed as

$$
P_{O}=\sum_{l=1}^{L} \sum_{\hat{l}=1}^{L} \boldsymbol{P}_{\text {sel }}^{\hat{B}}(l, \hat{l})\left[\frac{\gamma\left(m_{l}, \frac{m_{l} \gamma_{T_{l}}}{\bar{\gamma}_{l}}\right)}{\Gamma\left(m_{l}\right)}\right]^{\hat{l}} .
$$

\section{Diversity Order}

In a single hop, each $\bar{Q}_{l}\left(\sqrt{A_{i, j} \bar{\gamma}_{l}}\right)$ has the same $\bar{\gamma}_{l}$ and $m_{l}$, hence, $\bar{Q}_{l}\left(\sqrt{A_{i, j} \bar{\gamma}_{l}}\right)$ has the same diversity gain as $P_{L e}^{(l)}$. The diversity order $D O_{l}$ can be derived for the $l$ th hop from (18), yielding

$$
\begin{aligned}
\bar{Q}_{l}\left(\sqrt{A_{i, j} \bar{\gamma}_{l}}\right)= & \left(\frac{m_{l}}{\bar{\gamma}_{l}}\right)^{m_{l}} \frac{L}{\Gamma\left(m_{l}\right)} \int_{0}^{\infty} Q\left(\sqrt{A_{i, j} \gamma}\right) \gamma^{m_{l}-1} \\
& \times\left[\frac{\gamma\left(m_{l}, \frac{m_{l} \gamma}{\bar{\gamma}_{l}}\right)}{\Gamma\left(m_{l}\right)}\right]^{L-1} \exp \left(-\frac{m_{l} \gamma}{\bar{\gamma}_{l}}\right) d \gamma
\end{aligned}
$$

When $\bar{\gamma}_{l}$ is large, the incomplete gamma function can be approximated as [34] $(06.06 .26 .0002 .01)$

$$
\begin{aligned}
\gamma\left(m_{l}, \frac{m_{l} \gamma}{\bar{\gamma}_{l}}\right) / \Gamma\left(m_{l}\right) & =\frac{\left(\frac{m_{l} \gamma}{\bar{\gamma}_{l}}\right)^{m_{l}}}{\Gamma\left(m_{l}\right) m_{l}} \sum_{n=0}^{\infty} \frac{m_{l}}{m_{l}+n} \frac{\left(\frac{-m_{l} \gamma}{\bar{\gamma}_{l}}\right)^{n}}{n !} \\
& \approx \frac{1}{\Gamma\left(m_{l}\right) m_{l}}\left(\frac{m_{l} \gamma}{\bar{\gamma}_{l}}\right)^{m_{l}} .
\end{aligned}
$$

Then, substituting (46) into (45), we arrive at:

$$
\begin{aligned}
& \bar{Q}_{l}\left(\sqrt{A_{i, j} \bar{\gamma}_{l}}\right) \\
\approx & \frac{m_{l}^{m_{l} L-L+1}}{\bar{\gamma}_{l}^{m_{l} L}} \frac{L}{\Gamma\left(m_{l}\right)^{L}} \int_{0}^{\infty} Q\left(\sqrt{A_{i, j} \gamma}\right) \gamma^{m_{l} L-1} \exp \left(-\frac{m_{l} \gamma}{\bar{\gamma}_{l}}\right) d \gamma \\
\approx & \frac{m_{l}^{m_{l} L-L+1}}{\bar{\gamma}_{l}^{m_{l} L}} \frac{L}{\Gamma\left(m_{l}\right)^{L}} \frac{1}{2 \sqrt{\pi} m_{l} L}\left(\frac{2}{A}\right)^{m_{l} L} \Gamma\left(m_{l} L+\frac{1}{2}\right) \\
= & C\left(\frac{1}{\bar{\gamma}_{l}^{m_{l}}}\right)^{L} .
\end{aligned}
$$

where $C$ is not related to $\bar{\gamma}_{l}$. From (47) we can explicitly see that the achievable diversity order of the lth hop is $D O_{l}=L$, when there are $L$ available hops for activation. Furthermore, according to the operational principles of the MHD, the endto-end performance of the MHL also benefits from attaining the diversity order of $D O_{l}=L$.

Note that the delay analysis of the MHLs employing the proposed MHD scheme is identical to that in [16]. More explicitly, the corresponding probability mass function (PMF) of the delay and the average delay of the system are the same as those in [16], but we have to replac $B$ in [16] by the equivalent buffer size of $\hat{B}=B / \log _{2} M$. Again, similarly to [16], the maximum and minimum packet delay are given by $\hat{B} L^{2}-\hat{B} L-L+2$ and $L$ TSs. Finally the distribution of the delay is given by (36) of [16]. Given the distribution of the delay, the average delay may be readily calculated. The average delay increases with the number of hops and/or the size $\hat{B}$ of the buffer. The corresponding examples can be found in Fig. 10 and Fig.11 of [16].

\section{Performance Results}

In this section, we provide a range of performance results, in order to characterize both the BER and outage performance of the MHLs employing our MHD scheme, when communicating over Nakagami- $m$ fading channels. We illustrate the effects of various MQAM schemes, buffer sizes, distances of the different hops and of different Nakagami- $m$ fading parameters 


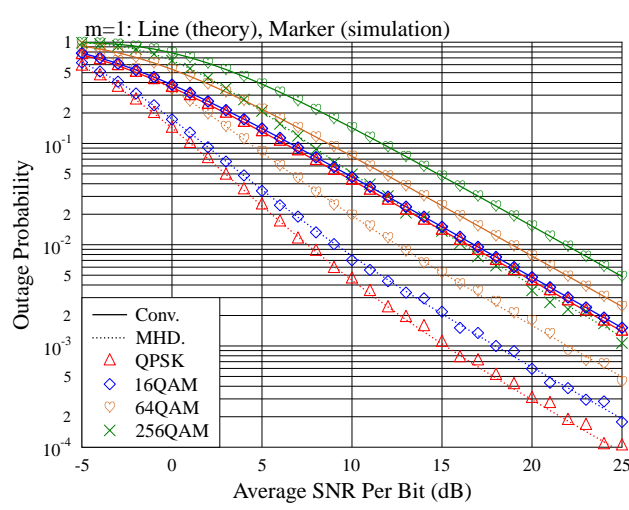

Fig. 11. Outage performance of two-hop links with different modulation schemes, when communicating over Rayleigh $(m=1)$ fading channels. The buffer size of every RN is $B=32$ packets, the modulation scheme is 16QAM and both hops have the same distance.

our multihop links, when various MQAM schemes, various buffer sizes and various number of hops are considered. These figures stipulate the same assumptions, as Figs. 4, 7 and 8, respectively. Note that in our numerical computations and simulations, the threshold set of $\gamma_{T_{1}}=\cdots=\gamma_{T_{L}}=\gamma_{T}$ was adjusted for maintaining a BER of 0.03 for a single-hop link, when the received energy for a bit transmitted from the $\mathrm{SN}$ to DN was 1 [35]. The outage probability of the corresponding conventional multihop scheme is also provided for the sake of comparison in Fig. 9 and Fig. 11. From these figures, we may derive similar observations to those emerging from Figs. 4, 7 and 8. In summary, in Fig. 9, a significant multihop diversity gain is observed for the RNs employing buffers of a sufficiently high size. The line with solid circles, which represents the case of a large buffer size $(B=512$ packets giving an equivalent buffer size of $\hat{B}=128$ ), approaches the theoretical bound. Fig. 10 shows that the outage probability reduces, as the number of hops increases. Fig. 11 characterize the outage probability of two hop links relying on different modulation scheme, as evaluated from (30) and (44), which exhibits a lower improvement for higher-order modulation schemes, because the equivalent buffer size of higher-order modulation schemes is lower than for lower-order modulation schemes.

\section{Conclusions}

In this contribution, a multihop transmission scheme, namely the MHD scheme, was proposed for transmission of information over MHLs. Within a given TS, our MHD scheme activates a specific hop from the set of the all available hops based on the values given by their CDFs. Our analysis showed that this CDF-aware MHD scheme represents a generalized MHD scheme, which is suitable for general MHLs, where different hops may have different average SNRs and experience different types of fading CDFs. Furthermore, our MHD scheme is capable of achieving the full diversity gain provided by the independent fading experienced by different hops. In this paper, the performance of MHLs employing the MHD scheme was investigated, when communicating over Nakagami- $m$ fading channels. Both accurate and approximate expressions were derived for the end-to-end BER as well as for the outage probability of BPSK/MQAM signals. Furthermore, the attainable diversity order was also derived. Our performance results showed that exploiting the independent fading of different hops results in a significant diversity gain. In general, the achievable multihop diversity gain increases, as the relay's buffer size increases as well as when the affordable packet delay increases. The achievable multihop diversity gain improved as the number of hops increased. Therefore, the MHD-assisted multihop transmission is significantly more energy-efficient than the conventional multihop transmission, although both of them have higher energy-efficiency than single-hop transmissions.

In our future research, adaptive modulation and coding (AMC) will be integrated into our CDF-aware MHD scheme.

\section{ACKNOWLEDGMENT}

The authors would like to thank the anonymous reviewers for their valuable comments and suggestions to improve the quality of the paper.

\section{REFERENCES}

[1] M. Mardani, J. S. Harsini, F. Lahouti, and B. Eliasi, "Joint adaptive modulation-coding and cooperative ARQ for wireless relay networks,", in IEEE International Symposium on Wireless Communication Systems. ISWCS '08., pp. 319 -323, Oct. 2008.

[2] D. Wubben, "High quality end-to-end-link performance," IEEE Vehicular Technology Magazine, vol. 4, no. 3, pp. 26 -32, Sept. 2009.

[3] A. Muller and H.-C. Yang, "Dual-hop adaptive packet transmission systems with regenerative relaying," IEEE Transactions on Wireless Communications, vol. 9, no. 1, pp. 234 -244, Jan. 2010.

[4] V. Asghari, A. Maaref, and S. Aissa, "Symbol error probability analysis for multihop relaying over Nakagami fading channels," " in IEEE Wireless Communications and Networking Conference. WCNC'10, pp. $1-6$, Apr. 2010.

[5] M. Hasna and M.-S. Alouini, "End-to-end performance of transmission systems with relays over Rayleigh-fading channels," IEEE Transactions on Wireless Communications, vol. 2, no. 6, pp. 1126 - 1131, Nov. 2003.

[6] - "Harmonic mean and end-to-end performance of transmission systems with relays," IEEE Transactions on Communications, vol. 52, no. 1, pp. 130 - 135, Jan. 2004.

[7] — "Outage probability of multihop transmission over Nakagami fading channels," IEEE Communications Letters, vol. 7, no. 5, pp. 216 - 218, May 2003.

[8] J. Cao, L.-L. Yang, and Z. Zhong, "Performance of multihop wireless links over generalized-K fading channels," " in IEEE 72nd Vehicular Technology Conference Fall. VTC 2010-Fall, pp. 1 -5, Sept. 2010.

[9] G. Karagiannidis, T. Tsiftsis, and R. Mallik, "Bounds for multihop relayed communications in Nakagami-m fading," IEEE Transactions on Communications, vol. 54, no. 1, pp. 18 - 22, Jan. 2006.

[10] G. Karagiannidis, "Performance bounds of multihop wireless communications with blind relays over generalized fading channels," IEEE Transactions on Wireless Communications, vol. 5, no. 3, pp. 498 - 503, Mar. 2006

[11] X. Zhang and Y. Gong, "Adaptive power allocation for regenerative relaying with multiple antennas at the destination," IEEE Transactions on Wireless Communications, vol. 8, no. 6, pp. 2789 -2794, June 2009.

[12] Z. Lin, E. Erkip, and M. Ghosh, "Rate adaptation for cooperative systems," " in IEEE Global Telecommunications Conference. GLOBECOM '06., pp. 1 -5, Dec. 2006.

[13] A. Ikhlef, D. Michalopoulos, and R. Schober, "Max-max relay selection for relays with buffers," IEEE Transactions on Wireless Communications, vol. 11, no. 3, pp. $1124-1135$, March 2012.

[14] — "Buffers improve the performance of relay selection," " in 2011 IEEE Global Telecommunications Conference, GLOBECOM 2011, pp. $1-6$, Dec. 2011.

[15] C. Dong, L.-L. Yang, and L. Hanzo, "Multihop diversity for fading mitigation in multihop wireless networks," " in 2011 IEEE Vehicular Technology Conference (VTC Fall), pp. 1 -5, Sept. 2011. 
[16] — - "Performance analysis of multi-hop diversity aided multi-hop links," IEEE Transactions on Vehicular Technology, vol. 61, no. 6, pp. $2504-2516,2012$.

[17] L.-L. Yang, C. Dong, and L. Hanzo, "Multihop diversity-a precious source of fading mitigation in multihop wireless networks," "in IEEE Global Telecommunications Conference, 2011. GLOBECOM '11., 2011.

[18] J. Boyer, D. Falconer, and H. Yanikomeroglu, "Multihop diversity in wireless relaying channels," IEEE Transactions on Communications, vol. 52, no. 10, pp. 1820 - 1830, Oct. 2004.

[19] L. Yang, M. Hasna, and M.-S. Alouini, "Average outage duration of multihop communication systems with regenerative relays," IEEE Transactions on Wireless Communications, vol. 4, no. 4, pp. 1366 1371 , July 2005.

[20] M. Nakagami, "The m-distribution, a general formula of intensity of rapid fading," Statistical Methods in Radio Wave Propagation: Proceedings of a Symposium, vol. 47, pp. 3-36, June 1958.

[21] I. Gradshteyn and I. Ryzhik, Table of Integrals, Series, and Products, Seventh Edition. Elsevier Pte Ltd., 2007.

[22] M. Matthaiou, D. Laurenson, and J. Thompson, "A MIMO Channel Model Based on the Nakagami-Faded Spatial Eigenmodes," IEEE Transactions on Antennas and Propagation,, vol. 56, no. 5, pp. 1494-1497, 2008.

[23] J. Kermoal, L. Schumacher, K. Pedersen, P. Mogensen, and F. Frederiksen, "A stochastic MIMO radio channel model with experimental validation," IEEE Journal on Selected Areas in Communications, vol. 20, no. 6, pp. 1211-1226, 2002.

[24] W. Weichselberger, "Spatial structure of multiple antenna radio channels: a signal processing viewpoint," Ph.D. dissertation, Technical Univ. Vienna, Austria, Dec 2003.

[25] M. K. Simon and M. S. Alouini, Digital Communication over Fading Channels:A unified Approach to Performance Analysis. Wiley-IEEE Press, Apr. 2000

[26] M.-S. Alouini and M. Simon, "Performance of coherent receivers with hybrid SC/MRC over Nakagami-m fading channels," IEEE Transactions on Vehicular Technology, vol. 48, no. 4, pp. 1155 -1164, July 1999.

[27] L. Hanzo, S. Ng, W. Webb, and T. Keller, Quadrature Amplitude Modulation: From Basics to Adaptive Trellis-Coded, Turbo-Equalised and Space-Time Coded OFDM, CDMA and MC-CDMA Systems. IEEE Press-John Wiley, September 2004. [Online]. Available: http://eprints.soton.ac.uk/260296/

[28] L.-L. Yang and L. Hanzo, "A recursive algorithm for the error probability evaluation of m-qam," IEEE Communications Letters, vol. 4, no. 10, pp. $304-306$, Oct. 2000.

[29] G. Golub and C. Loan, Matrix Computations, 3rd ed. The Johns Hopkins University Press, Apr. 1996.

[30] T. Eng and L. Milstein, "Coherent ds-cdma performance in Nakagami multipath fading," IEEE Transactions on Communications, vol. 43, no. 234, pp. $1134-1143$, Feb. 1995.

[31] E. Morgado, I. Mora-Jimenez, J. Vinagre, J. Ramos, and A. Caamano, "End-to-End average ber in multihop wireless networks over fading channels," IEEE Transactions on Wireless Communications, vol. 9, no. 8, pp. $2478-2487$, Aug. 2010.

[32] D. Bertsekas and R. Gallager, Data Networks, 2nd ed. Prentice-Hall, Apr. 1992.

[33] R. Horn and C. Johnson, Matrix Analysis. Cambridge University Press New York, NY, USA, Apr. 1986.

[34] http://functions.wolfram.com/.

[35] B. J. Choi and L. Hanzo, "Optimum mode-switching-assisted constantpower single- and multicarrier adaptive modulation," IEEE Transactions on Vehicular Technology, vol. 52, no. 3, pp. 536 - 560, May 2003.

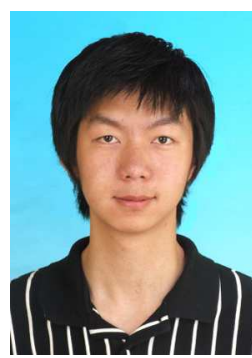

Chen Dong received his BS degree in electronic information sciences and technology from University of Science and Technology of China(USTC), Hefei, China in 2004, and his MEng degree in pattern recognition and automatic equipment from the Graduate University of Chinese Academy of Sciences(GUCAS), Beijing, China in 2007. Since 2009, he has been working towards his $\mathrm{PhD}$ degree with the Communications, Signal Processing and Control (CSPC) group, School of Electronics and Computer Science, University of Southampton, UK. He is the recipient of scholarship under the
UK-China Scholarships for Excellence programme. His research interests include applied math, relay system, channel modelling and cross-layer optimization.

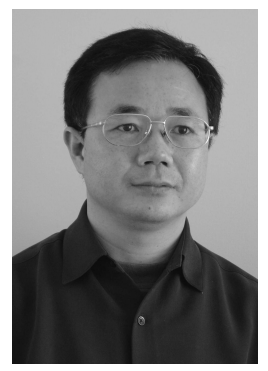

Lie-Liang Yang (M'98, SM'02) received his BEng degree in communications engineering from Shanghai TieDao University, Shanghai, China in 1988, and his MEng and PhD degrees in communications and electronics from Northern (Beijing) Jiaotong University, Beijing, China in 1991 and 1997, respectively. From June 1997 to December 1997 he was a visiting scientist of the Institute of Radio Engineering and Electronics, Academy of Sciences of the Czech Republic. Since December 1997, he has been with the University of Southampton, United Kingdom, where he is the professor of wireless communications in the School of Electronics and Computer Science. Dr. Yang's research has covered a wide range of topics in wireless communications, networking and signal processing. $\mathrm{He}$ has published over 270 research papers in journals and conference proceedings, authored/co-authored three books and also published several book chapters. He is currently an associate editor to the IEEE Trans. on Vehicular Technology, Journal of Communications and Networks (JCN) and the Security and Communication Networks (SCN) Journal.

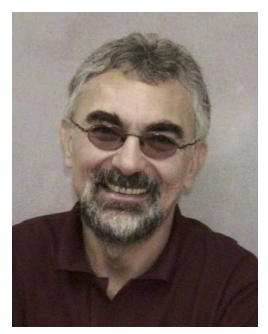

Lajos Hanzo FREng, FIEEE, FIET, Fellow of EURASIP, DSc received his degree in electronics in 1976 and his doctorate in 1983. In 2009 he was awarded the honorary doctorate "Doctor Honoris Causa" by the Technical University of Budapest. During his 37-year career in telecommunications he has held various research and academic posts in Hungary, Germany and the UK. Since 1986 he has been with the School of Electronics and Computer Science, University of Southampton, UK, where he holds the chair in telecommunications. He has successfully supervised $80+$ PhD students, co-authored 20 John Wiley/IEEE Press books on mobile radio communications totalling in excess of 10 000 pages, published $1300+$ research entries at IEEE Xplore, acted both as TPC and General Chair of IEEE conferences, presented keynote lectures and has been awarded a number of distinctions. Currently he is directing a 100-strong academic research team, working on a range of research projects in the field of wireless multimedia communications sponsored by industry, the Engineering and Physical Sciences Research Council (EPSRC) UK, the European Research Council's Advanced Fellow Grant and the Royal Society's Wolfson Research Merit Award. He is an enthusiastic supporter of industrial and academic liaison and he offers a range of industrial courses. He is also a Governor of the IEEE VTS. During 2008 2012 he was the Editor-in-Chief of the IEEE Press and a Chaired Professor also at Tsinghua University, Beijing. His research is funded by the European Research Council's Senior Research Fellow Grant. For further information on research in progress and associated publications please refer to http://www-mobile.ecs.soton.ac.uk Lajos has $18000+$ citations. 